ARTICLE

DOI: $10.1038 / \mathrm{s} 41467-018-03401-\mathrm{x}$

\title{
Three-dimensional localization of nanoscale battery reactions using soft X-ray tomography
}

Young-Sang Yu ${ }^{1,2}$, Maryam Farmand ${ }^{1}$, Chunjoong Kim²,3, Yijin Liu (i) 4, Clare P. Grey ${ }^{5,6}$, Fiona C. Strobridge ${ }^{5}$, Tolek Tyliszczak', Rich Celestre (1) ${ }^{1}$, Peter Denes ${ }^{1}$, John Joseph ${ }^{7}$, Harinarayan Krishnan (1) ${ }^{8}$, Filipe R.N.C. Maia9, A.L.David Kilcoyne (10 ${ }^{1}$, Stefano Marchesini ${ }^{1}$, Talita Perciano Costa Leite $\mathbb{D}^{8}{ }^{8}$, Tony Warwick ${ }^{1}$, Howard Padmore ${ }^{1}$, Jordi Cabana (i) ${ }^{2} \&$ David A. Shapiro (i) ${ }^{1}$

Battery function is determined by the efficiency and reversibility of the electrochemical phase transformations at solid electrodes. The microscopic tools available to study the chemical states of matter with the required spatial resolution and chemical specificity are intrinsically limited when studying complex architectures by their reliance on two-dimensional projections of thick material. Here, we report the development of soft X-ray ptychographic tomography, which resolves chemical states in three dimensions at $11 \mathrm{~nm}$ spatial resolution. We study an ensemble of nano-plates of lithium iron phosphate extracted from a battery electrode at $50 \%$ state of charge. Using a set of nanoscale tomograms, we quantify the electrochemical state and resolve phase boundaries throughout the volume of individual nanoparticles. These observations reveal multiple reaction points, intra-particle heterogeneity, and size effects that highlight the importance of multi-dimensional analytical tools in providing novel insight to the design of the next generation of high-performance devices.

\footnotetext{
${ }^{1}$ Advanced Light Source, Lawrence Berkeley National Laboratory, Berkeley, CA 94720, USA. ${ }^{2}$ Department of Chemistry, University of Illinois at Chicago, Chicago, IL 60607, USA. ${ }^{3}$ Department of Materials Science and Engineering, Chungnam National University, Daejeon, Chungnam 305-764, South Korea. ${ }^{4}$ Stanford Synchrotron Radiation Lightsource, SLAC National Accelerator Laboratory, Menlo Park, CA 94025, USA. ${ }^{5}$ Department of Chemistry, University of Cambridge, Lensfield Road, Cambridge CB2 1EW, UK. ${ }^{6}$ Department of Chemistry, Stony Brook University, Stony Brook, NY 11794, USA. ${ }^{7}$ Engineering Division, Lawrence Berkeley National Laboratory, Berkeley, CA 94720, USA. ${ }^{8}$ Computational Research Division, Lawrence Berkeley National Laboratory, Berkeley, CA 94720, USA. ${ }^{9}$ Department of Cell and Molecular Biology, Uppsala University, Husargatan 3, 75124 Uppsala, Sweden. Correspondence and requests for materials should be addressed to J.C. (email: jcabana@uic.edu) or to D.A.S. (email: dashapiro@lbl.gov)
} 
$\mathrm{T}$ echniques capable of analyzing chemical states at high spatial resolution are essential for elucidating the complex phenomena at the nanoscale that underpin materials' properties. For example, battery function is determined by the efficiency and reversibility of the electrochemical phase transformations at solid electrodes, creating the need to accurately define relationships between chemistry, mechanics, and morphology ${ }^{1,2}$. Conventional X-ray imaging methods are well suited to probe chemical states in bulk matter, but they are also limited in spatial resolution to a few tens of nanometers by the Xray optics ${ }^{3-5}$. Furthermore, bulk X-ray diffraction can unambiguously differentiate between two-phase and metastable singlephase delithiation pathways, but it cannot map heterogeneities in the spatial distribution of such states ${ }^{6}$. In turn, electron-based techniques achieve very-high spatial resolution ${ }^{7-9}$ and can provide three-dimensional (3D) quantification of the chemical state $^{10}$, but they also suffer from diffraction contrast effects and non-linearities for material thicknesses greater than the meanfree-path of inelastic scattering. Soft X-ray ptychography has recently narrowed the gap in spatial resolution while retaining high sensitivity to chemical states and penetration through functional volumes of matter ${ }^{11,12}$. If data are only collected along one two-dimensional (2D) projection, the analysis of complex systems becomes problematic because of the likelihood of overlapping material with differing chemical components $s^{3,4}$. This problem is readily solved by the use of X-ray based computed tomography, but the quantification of chemical states in three dimensions by conventional methods comes with limited spatial resolution, which is currently, at best, $30 \mathrm{~nm}^{3,13-15}$.

Here, we have combined soft X-ray ptychographic imaging and computed spectro-tomography to determine the 3D morphology and oxidation states of transition-metal cations in agglomerated cathode nanoparticles of lithium iron phosphate $\left(\mathrm{LiFePO}_{4}\right)$ at 11 $\mathrm{nm}$ 3D spatial resolution. The measured absorption at each voxel and X-ray photon energy is converted to optical density (OD) and used for computing quantitative $3 \mathrm{D}$ chemical composition. We investigate the complex correlation between chemical phase distribution and morphology in single nano-plates of $\mathrm{LiFePO}_{4}$, a material that epitomizes the fundamental nature of intercalation chemistry that enables electrodes for high energy density Li-ion batteries ${ }^{16,17}$. The mechanism of transformation of $\mathrm{LiFePO}_{4}$ is one of the most intensely studied reactions in battery chemistry. While the reaction proceeds through a first-order transition in equilibrium ${ }^{16,18-20}$, under certain kinetic conditions, metastable pathways based on solid solutions have been observed ${ }^{21-24}$. These pathways bypass penalties in coherency strain due to the coexistence of phases in one particle, both enabling completion of the reaction and faster kinetics. The exact conditions that determine these pathways and, more generally, how electrochemical transformations can occur within single particles of battery electrodes are still widely debated topics. Our approach enabled both direct observation of the static internal chemical structure within crystals as small as $20 \mathrm{~nm}$ in their smallest dimension and the evaluation of correlations of the state of charge with particle size among a statistically significant number of particles.

\section{Results}

Sample synthesis and 3D chemical mapping. $\mathrm{LiFePO}_{4}$ nanoplates $\left(100 \times 80 \times 20 \mathrm{~nm}^{3}\right)$ were electrochemically delithiated in a Li metal half-cell until $50 \%$ of the total amount of lithium was extracted, based on coulometric analysis of the cell response (see Methods and Supplementary Figs. 1-2). The delithiation was conducted at a slow rate to maximize reaction homogeneity ${ }^{25}$ across the electrode and minimize the formation of metastable states that could relax during the harvesting of the particles ${ }^{22}$.
Coulometry is an adequate method to control the average composition of the electrode due to the absence of side reactions at the potentials of operation ${ }^{26}$. Indeed, bulk X-ray diffraction of the partly delithiated sample confirmed the co-existence of two phases (Supplementary Fig. 3). The position of the diffraction peaks of one phase were in agreement with $\mathrm{Li}_{\alpha} \mathrm{FePO}_{4}$, where $\alpha$ was slightly smaller than 1 , consistent with small domains of $\mathrm{Li}$ solubility observed in previous studies ${ }^{16,27,28}$. In contrast, the second phase showed peak positions comparable to $\mathrm{FePO}_{4}$, consistent with reports that the solubility of $\mathrm{Li}$ on the $\mathrm{Li}$-poor end of the phase diagram, $\beta$ in $\mathrm{Li}_{\beta} \mathrm{FePO}_{4}$, is very small ${ }^{27}$. Analysis of the relative intensities using methodologies of analysis in the literature ${ }^{29}$ confirmed the presence of these two phases at $\sim 50 \%$ ratio. The large facets of the plates correspond to the ac crystallographic plane with the long axis parallel to $c^{12,30}$.

Tomographic data from over 100 harvested particles were collected near the Fe $L_{3}$ edge at 708.2 and $710.2 \mathrm{eV}$ (Fig. 1a and Supplementary Movie 1), which correspond to the maxima of the absorption resonances for $\mathrm{LiFePO}_{4}\left(\mathrm{Fe}^{2+}\right)$ and $\mathrm{FePO}_{4}\left(\mathrm{Fe}^{3+}\right)$, respectively, as shown in Supplementary Fig. 5 and in the literature ${ }^{31-33}$. The 3D resolution of $11 \mathrm{~nm}$ was confirmed by Fourier shell correlation (FSC) and line-profiles (Fig. 1b-d and Supplementary Figs. 7-8). Note that the actual resolution should be somewhat higher as the FSC reduces the signal-to-noise ratio of the data by a factor of two at all spatial frequencies. The oxidation states of the individual nano-plates were quantified from measurements of the OD at only two energies ${ }^{13}, 34-36$ because, in three dimensions, thickness effects, which typically require measurement of off-resonant data for normalization can be neglected owing to the constant voxel size (see Methods). Thus, the chemical composition is computed directly from the polar angle in the correlation plot (Fig. 2a-b, see Methods). The distribution of compositions per voxel was clearly bimodal (centers of polar angle histogram: $\mathrm{Li}_{0.93} \mathrm{FePO}_{4}$ and $\mathrm{Li}_{0.02} \mathrm{FePO}_{4}$ ), in agreement with the bulk XRD measurement of the sample, yet the average state-of-charge (SOC) obtained by analyzing the $3 \mathrm{D}$ volume and independently measured 2D XAS data was $16.2 \%$ and $16.8 \%$, respectively (Supplementary Figs. 10-11). The apparent discrepancy between this value and the 50\% SOC of the electrochemical cell could occur if the population of particles harvested for the tomogram were located in a portion of the electrode with a deficiency in carbon content and/or electrolyte wetting, introducing transport deficiencies that delayed their reaction. The existence of two differentiated spectroscopic components is consistent with the presence of $\mathrm{Li}_{\alpha} \mathrm{FePO}_{4}(\alpha \geq$ $0.9)$ or $\mathrm{Li}_{\beta} \mathrm{FePO}_{4}(\beta<0.05)^{16}, 27,28$. Upon inspection of the resulting tomogram (Fig. $2 c$ and Supplementary Movie 1), these components were found to coexist within the same particle in several cases.

Chemical phase distributions of individual particles. In the case of unlimited 3D spatial resolution and a binomial distribution, the upper limit of the composition error can be defined by the root-mean-square (RMS) widths of each Gaussian distribution of compositions (Fig. $2 \mathrm{~b}$ and Methods), which are $\pm 11.3 \%$ for $\mathrm{Li}_{\alpha} \mathrm{FePO}_{4}\left(\mathrm{Fe}^{2+}\right.$-rich $)$ and $\pm 13.9 \%$ for $\mathrm{Li}_{\beta} \mathrm{FePO}_{4}\left(\mathrm{Fe}^{3+}\right.$-rich $)$. As a result, these measurements cannot resolve the small $\mathrm{Li}$ solubilities in the co-existing phases, revealed by XRD above, because they are smaller than the calculated error. Consequently, $\mathrm{Li}_{\alpha} \mathrm{FePO}_{4}$ is referred as LFP-rich, and $\mathrm{Li}_{\beta} \mathrm{FePO}_{4}$ as FP-rich. To reduce the impact of compositional error and enhance the resolution of domains that were chemically distinct, the voxels in the $3 \mathrm{D}$ map (Fig. 2c) were segmented into three major components, $>70 \%$ $\mathrm{Li}_{\alpha} \mathrm{FePO}_{4}$ (LFP-rich), $>70 \% \mathrm{Li}_{\beta} \mathrm{FePO}_{4}$ (FP-rich), and mixed (i.e., $30-70 \% \mathrm{Li}_{\alpha} \mathrm{FePO}_{4}$, the rest being $\left.\mathrm{Li}_{\beta} \mathrm{FePO}_{4}\right)$, with the 

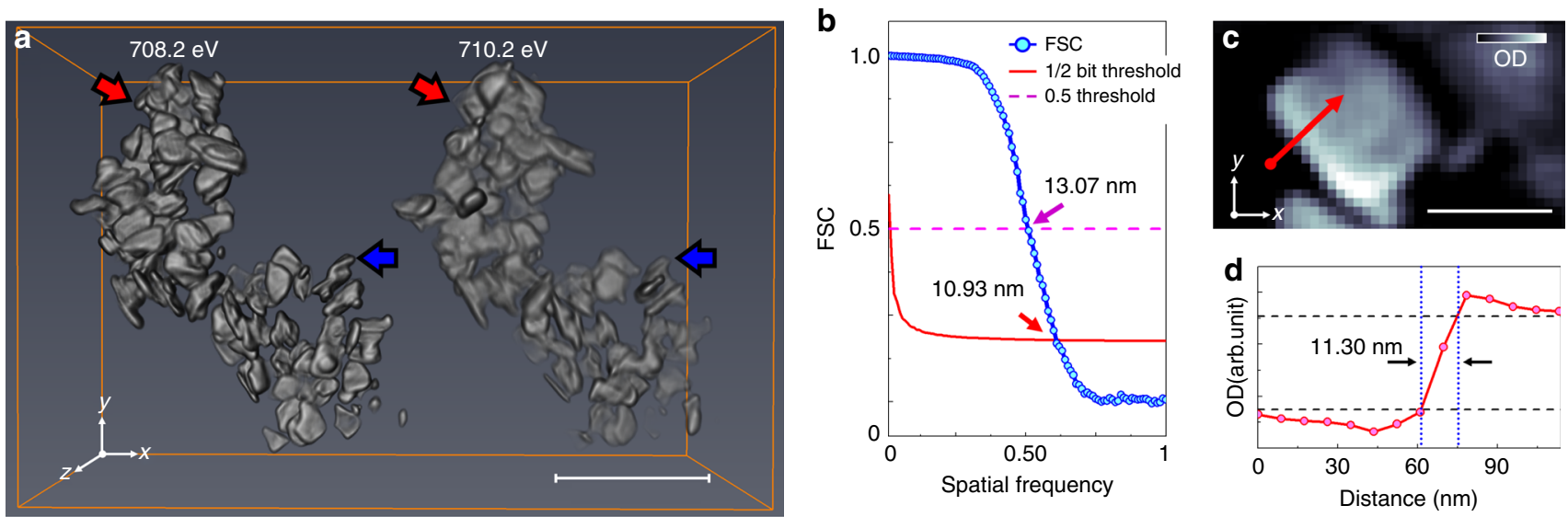

Fig. 1 Results of tomographic reconstruction. a Reconstructed three-dimensional (3D) optical density volumes at 708.2 (left) and 710.2 eV (right). The size of reconstructed voxels is $6.7 \times 6.7 \times 6.7 \mathrm{~nm}^{3}$. b Resolution estimation of the $3 \mathrm{D}$ volume at $708.2 \mathrm{eV}$ in $\mathbf{a}$ by Fourier shell correlation (FSC, blue solid line with scatter) with 1/2-bit (red solid line) and 0.5 (magenta dashed-line) threshold criteria. c Representative cross-section of the tomogram at $708.2 \mathrm{eV}$ along the highest resolution plane $(x y)$. The slice of the same position at $710.2 \mathrm{eV}$ is shown in Supplementary Fig. 7. The positions of the slices are marked as red (cutting along $x y$ plane) and blue (cutting along $x z$ plane) arrows in $\mathbf{a}$. The resultant cross-sections onto the lower resolution plane ( $x z$ plane) at both 708.2 and $710.2 \mathrm{eV}$ are shown in Supplementary Fig. 8. $\mathbf{d}$ Line profile indicated by the red arrow in c. Black-dashed lines are guides for 10-90\% resolution criteria. Scale bars in a and $\mathbf{c}$ indicate 500 and $100 \mathrm{~nm}$, respectvely
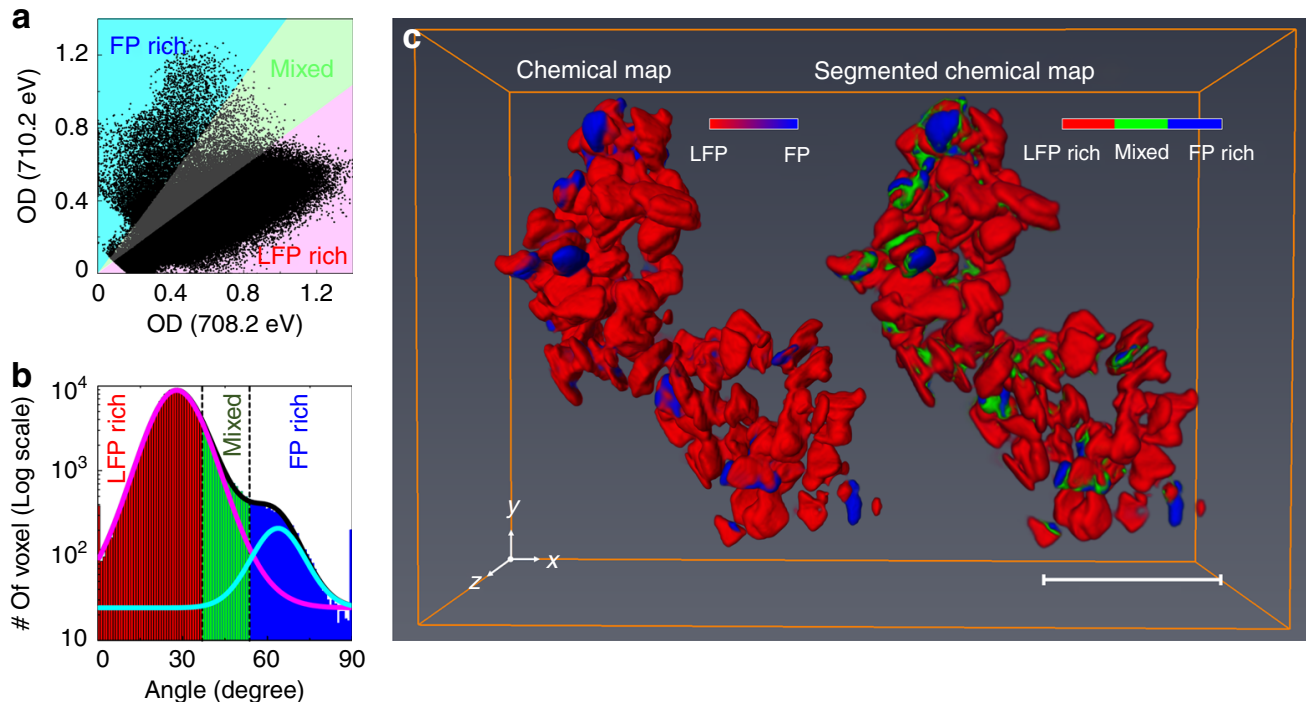

Fig. 2 Three-dimensional (3D) chemical state mapping. a Correlative distribution plots between the optical densities (ODs) of each voxel at 708.2 and $710.2 \mathrm{eV}$. b Histogram plot of the polar angles of the data points in $\mathbf{a}$. The $y$-axis is expressed as a logarithmic scale for better visibility. The plot can be fitted with summation (black solid line) of two Gaussian distributions which are centered on low (magenta solid line, $27.48^{\circ}$ ) and high (cyan solid line, $65.0^{\circ}$ ) polar angles correspond to $\mathrm{Li}_{0.93} \mathrm{FePO}_{4}$ and $\mathrm{Li}_{0.02} \mathrm{FePO}_{4}$, respectively. c $3 \mathrm{D}$ chemical map (left) and its segmentation into three chemical phase groups (right). The presence of the $\mathrm{Li}_{\alpha} \mathrm{FePO}_{4}$ (majority $\mathrm{Fe}^{2+}, \mathrm{LFP}$ ) and charged $\mathrm{Li}_{\beta} \mathrm{FePO}_{4}$ (majority $\mathrm{Fe}^{3+}, \mathrm{FP}$ ) were assigned colors red and blue, respectively (left). The voxels were separated into three distinct groups, indicating chemical phase group of each voxel, according to the polar angle. The red, green, and blue areas indicate LFP-rich $\left(>70 \% \mathrm{Li}_{\alpha} \mathrm{FePO}_{4}\right)$, FP-rich $\left(>70 \% \mathrm{Li}_{\beta} \mathrm{FePO}_{4}\right)$, and Mixed $\left(30-70 \% \mathrm{Li}_{\alpha} \mathrm{FePO}_{4}\right.$, the rest being $\left.\mathrm{Li}_{\beta} \mathrm{FePO}_{4}\right)$ domains, respectively. The shading colors in $\mathbf{a}, \mathbf{b}$ indicate the criteria for chemical segmentation. Scale bar, $500 \mathrm{~nm}$

segmentation threshold at $30 \%$ (Supplementary Methods). It is important to emphasize that this segmentation purely reflects a conservative limit of detection of a given phase, and not its specific composition (e.g., $\alpha$ in $\mathrm{Li}_{\alpha} \mathrm{FePO}_{4}$ ). It gives a clear view of the most reliable information and is in agreement with a similarly segmented 2D XAS map with a total error of $7.4 \%$ (Supplementary Fig. 12). A total of 83 individual particles were segmented as shown in Fig. 3a. The fraction of particles with smaller dimensions in the tomogram was found to be higher in comparison with a larger, and, thus, more representative population of pristine particles imaged by transmission electron microscopy
(TEM, Supplementary Fig. 13). Though similarly shaped, the particles presented a variety of delithiation patterns, volumes, and total composition. A histogram of particle volumes and the corresponding fraction of activated particles is shown in Fig. 3b. The average composition of all voxels in each of the morphologically segmented particles was considered to determine particle activity. Active particles showed a statistically significant level of delithiation, defined as $15-100 \% \mathrm{Li}_{\beta} \mathrm{FePO}_{4}$ based on a compositional error threshold $(\sim 13 \%)$. This activity threshold should both encompass our composition error and avoid misinterpretation of particles as active due to the solubility limit of $\mathrm{Li}_{\beta} \mathrm{FePO}_{4}$. While, 

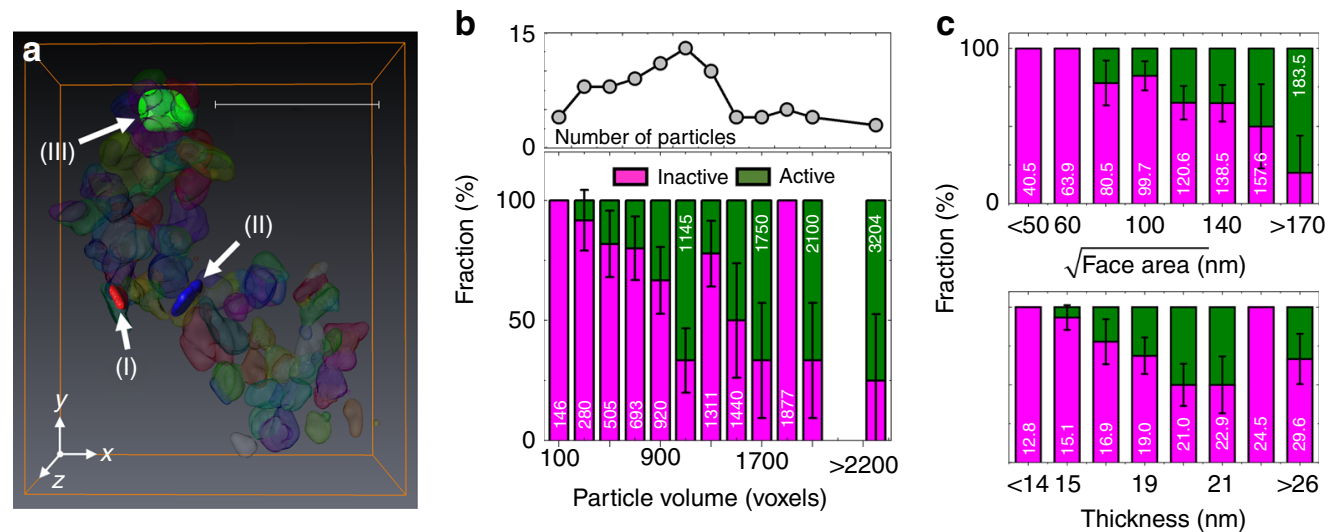

Fig. 3 Activeness of each particle. a Voxel segmentation to define individual particles. Scale bar, $500 \mathrm{~nm}$. b Volume distributions (black solid line with scatter) of individual particles shown in Fig. 2 and the fraction (bar plot) of inactive (magenta bar, $<15 \%$ of $\mathrm{Li}_{\beta} \mathrm{FePO}_{4}$ ) and active (olive bar, $15-100 \%$ of $\mathrm{Li}_{\beta} \mathrm{FePO}_{4}$ ) particles as a function of particle volume with an increment of 200 voxels' volume. Averaged particle volume in each range also shown. Each voxel has a volume of $6.7 \times 6.7 \times 6.7 \mathrm{~nm}^{3}$. The experimental error bars are calculated assuming a binomial distribution (active or inactive) taken at one standard deviation. c Compositional analysis based on the dimensions of each plate, comparing the facet area with thickness. The optical densities (ODs) of voxels along the particle thickness direction were averaged out across whole large facet. The thickness of the particle was calculated by the full-widthhalf-maxima of the averaged OD. The bar plots have the same color definition as $\mathbf{b}$

overall, $\mathrm{Li}_{\alpha} \mathrm{FePO}_{4}$ was the major component across the range of particle volumes, 25 particles $(30.1 \pm 5.1 \%)$ were defined as active if they had more than $15 \% \mathrm{Li}_{\beta} \mathrm{FePO}_{4}$. Even with a tighter error threshold $\left(25-100 \% \mathrm{Li}_{\beta} \mathrm{FePO}_{4}\right), 12.1 \pm 3.6 \%$ particles are categorized as active. The error range is calculated assuming a binomial distribution (active or inactive) taken at one standard deviation. Only one of the (partly) delithiated particles was found to be close to the oxidized state $\left(>70 \% \mathrm{Li}_{\beta} \mathrm{FePO}_{4}\right)$. A weak tendency was observed toward an increase in the population of active particles with volume (Fig. 3b). However, the variation in $a c$ facet area $\left(2500 \sim 40,000 \mathrm{~nm}^{2}\right)$ was much larger than particle thickness $(10 \sim 30 \mathrm{~nm})$. Tomography enabled the separate analysis of these particle dimensions, as shown in Fig. 3c. Interestingly, a significantly stronger dependence of the number of activated particles with facet size was found compared to overall volume. The averaged $\mathrm{Li}_{\alpha} \mathrm{FePO}_{4}$ concentrations for each individual particle showed a similar trend with respect to the facet area (Supplementary Fig. 14). Essentially no trend was found with particle thickness, likely due to the combination of a narrow distribution of values in this dimension and the small number of significant voxels along it due to finite spatial resolution. The fact that particles with small facets were both systematically found to be closer to the reduced state (Fig. 3c) and oversampled in the tomogram compared to the overall population of the electrode (Supplementary Fig. 13) could also explain the discrepancy between the overall SOC in the 3D image and the electrochemical cell.

Representative 3D chemical phase distributions for individual particles are shown in Fig. 4. These particles were chosen because their thickness was high enough compared to the spatial resolution to lead to insight into chemical gradients in all 3D. They also represent different degrees of delithiation. The particle showing the lowest degree of delithiation (Fig. $4 \mathrm{a}-\mathrm{c}$, overall composition $\mathrm{Li}_{0.89} \mathrm{FePO}_{4}$ ) showed evidence of reaction primarily around the edges (blue arrows) and along one of the two large facets (red arrows) of the crystal, without clear crystallographic directionality. The existence of multiple points of reaction propagation was confirmed in a second particle at a higher delithiation state (Fig. $4 \mathrm{~d}-\mathrm{f}$, overall composition $\mathrm{Li}_{0.81} \mathrm{FePO}_{4}$ ). In this case, delithation was also found to have occurred (red arrows) through the entire thickness of the particle. In contrast, a sharp division between single, large lithiated, and delithiated domains was observed in a particle at a much higher state of delithiation (Fig. 4g-i, overall composition $\mathrm{Li}_{0.41} \mathrm{FePO}_{4}$ ).

\section{Discussion}

The 3D snapshot of partly reacted states revealed the existence of sharp inhomogeneities within a particle, ascribed to co-existence of $\mathrm{Li}_{\alpha} \mathrm{FePO}_{4}$ or $\mathrm{Li}_{\beta} \mathrm{FePO}_{4}$, where $\alpha \gg \beta$ and $(\alpha-\beta)$ defines the miscibility gap. The exact values of $\alpha$ and $\beta$ could not be extracted at the chemical resolution of our measurement. Phase coexistence has been observed in nanoparticles by others ${ }^{12}, 18,37,38$. It has been shown to prevail during slow charging in recent operando X-ray studies ${ }^{36,39,40}$. This intra-particle heterogeneity, in ex-situ conditions, is inconsistent with a models of dominocascade delithiation ${ }^{19}$ or relaxation from a kinetic solid solution state through inter-particle exchange of $\mathrm{Li}^{21,41,42}$, where populations of particles containing only either $\mathrm{Li}_{\alpha} \mathrm{FePO}_{4}$ or $\mathrm{Li}_{\beta} \mathrm{FePO}_{4}$ would be expected. The population of particles with detected heterogeneity is generally larger than in other studies ${ }^{19,34,43,44}$. It is plausible that the higher spatial resolution achieved with ptychography improved the detection of heterogeneity. It is also worth noting that the average sizes in these previous studies were well above $100 \mathrm{~nm}$, and, thus, much larger than here. Interestingly, Brunetti et al. reported that mixed particles were among the smallest within the studied population ${ }^{45}$. Particle-by-particle models of transformation ${ }^{44}$ would not account for the large population of the particles with at least $15 \%$ of delithiated phase (i.e., active) observed in this study $(30.1 \pm 5.1 \%)$. In contrast, this value was in good agreement with recent operando $\mu$-XRD, where an average $22 \%$ active particles was reported during a charge at $\mathrm{C} / 5^{40}$, adding support to the relevance of the tomographic observations here to working conditions.

The distribution of delithiated domains in individual particles was complex and irregular irrespective of their individual $\mathrm{Li}$ content. Complex patterns of delithiation in $\mathrm{LiFePO}_{4}$ nanoparticles have been recently predicted by Welland et al. using a thermodynamic 3D model, where the effect of coherency strain, elastic moduli, and surface wetting was considered ${ }^{46}$. Kinetically, such patterns could be aggravated by the existence of uneven electrical contacts within individual nanoparticles in a composite porous electrode, driven by local heterogeneities in component 

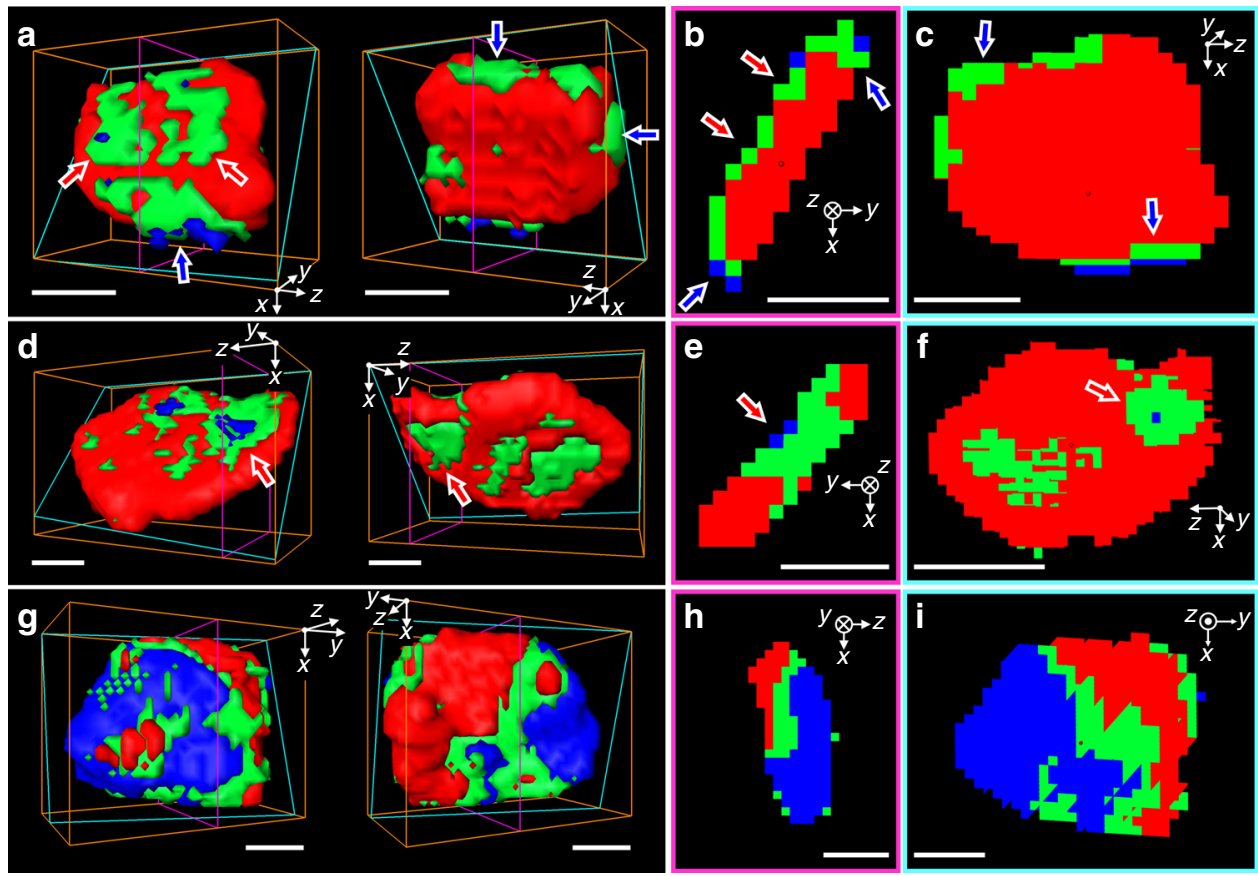

Fig. 4 Representative three-dimensional (3D) chemical phase distribution of individual particle. a, d, $\mathbf{g}$, Front (left) and backside (right) views of isosurface of three chemical components. Cross-sectional views along the thickness direction $(\mathbf{b}, \mathbf{e}, \mathbf{h})$ and along the large face (c, $\mathbf{f}, \mathbf{i})$, respectively. The cross-section planes are indicated as magenta and cyan colored boxes in 3D isosurface plots. The red, green, and blue indicate LFP-rich, mixed, and FP-rich voxels, respectively. The positions of each particle are noted as (I), (II), and (III) in Fig. 3a for $\mathbf{a}$, $\mathbf{d}$ and $\mathbf{g}$, respectively. All scale bars, $50 \mathrm{~nm}$

distribution. These contacts act as sinks of ions and electrons, and, thus, points of reaction initiation. If transient $\mathrm{Li}_{x} \mathrm{FePO}_{4}$ solid solutions were present under working conditions because phase separation is suppressed by coherency strain in these small particles $^{21,47}$, a heterogeneous distribution of electrical contacts, and, thus, carrier diffusion, could impose gradients in $x$ within a particle. Such gradients were recently predicted to trigger immediate spinodal decomposition into two phases even under applied current ${ }^{48}$, which would be consistent with our ex-situ observations. In a scenario where reaction progression is conditioned by electrical contacts, the correlation between large ac facets and increased level of delithiation found over many particles, also suggested by others ${ }^{46}$, could result from the larger probability of creating multiple points of electrical contact as facet area increases. Thermodynamic origins, imposed by coherency strain along the $a c$ facet, are also possible. Calculations of energy barriers to phase co-existence in $\mathrm{a} \mathrm{Li}_{x} \mathrm{FePO}_{4}$ nanoparticle available in the literature are conflicting. The $3 \mathrm{D}$ model by Welland et al. led to an inverse relationship with size $e^{46}$, which would predict larger nanoparticles reacting first, as observed here. In contrast, Cogswell et al. found lower or comparable barriers with decreasing size using a $2 \mathrm{D}$ model of the ac facet with depth averaging ${ }^{49}$. The discrepancy could, at least in part, be due to the different interfacial orientations in the two models, suggesting that further computational work would be helpful to establish this question.

Our demonstration of soft X-ray ptychographic tomography visualizes chemical states at a spatial resolution of $11 \mathrm{~nm}$ and is a powerful tool now available to chemists and materials scientists seeking insight into heterogenous chemical distributions occurring in $3 \mathrm{D}$, even in nanocrystals within relatively large fields view. This feature provides the opportunity to analyze ensemble statistics. Resolving phenomena in $3 \mathrm{D}$, at high resolution, allowed us to discern the effect of the dimensionality of anisotropies in transport and electrochemical reactivity. The combination with soft X-ray spectroscopic methods enables the analysis of chemical states in a variety of elements, from transition metals to common anions such as $O$. The resulting specimen thicknesses and required speeds are prohibitive for equivalent, high spatial resolution techniques in electron microscopy, making it an ideal complement to uncover phenomena at multiple scales in one measurement. This multiscale insight is critical to accurately defining the properties of functional materials in realistic architectures such as batteries.

\section{Methods}

Synthesis of $\mathrm{Li}_{\mathbf{x}} \mathbf{F e P O}_{4}$ (x $\left.\sim \mathbf{0 . 5}\right)$ nano-plates. Plate-shaped $\mathrm{LiFePO}_{4}$ were synthesized using a previously reported solvothermal method ${ }^{12,30} \cdot \mathrm{H}_{3} \mathrm{PO}_{4}$ and $\mathrm{LiOH} \cdot \mathrm{H}_{2} \mathrm{O}$ were dissolved in ethylene glycol at $50^{\circ} \mathrm{C}$, followed by addition of $\mathrm{FeSO}_{4} \cdot 7 \mathrm{H}_{2} \mathrm{O}$, for a total 1:1.5:2.7 molar ratio. After stirring for $30 \mathrm{~min}$, the mixture was heated at $180{ }^{\circ} \mathrm{C}$ for $10 \mathrm{~h}$. In order to achieve good conductivity, $\mathrm{LiFePO}_{4}$ nano-plates were carbon coated by mixing with $20 \mathrm{wt} \%$ of sucrose and then carbonizing at $650^{\circ} \mathrm{C}$ for $3 \mathrm{~h}$ in Ar atmosphere ${ }^{12,30}$. Powder X-ray diffraction patterns (Supplementary Fig. 3) were collected using a Bruker D8 Discover X-ray diffractometer operating with $\mathrm{Cu} K \alpha$ radiation $\left(\lambda_{\mathrm{avg}}=1.5418 \AA\right.$ ). They were consistent with mixtures of $\mathrm{LiFePO}_{4}$ (JCPDS card number 40-1499) and $\mathrm{FePO}_{4}$ (JCPDS card number 29-0715). In order to evaluate the macroscopic electrochemical properties of the oxide and prepare the specific state-of-charge $(\sim 50 \%)$, composite electrode films were fabricated by mixing the pristine oxide with acetylene black and polyvinylidene difluoride (PVDF) in a 80:10:10 ratio in $N$ methylpyrrolidone. The resulting slurry was cast onto a pre-weighed $\mathrm{Al}$ foil disk, dried at room temperature, followed by a heat treatment of $120^{\circ} \mathrm{C}$ under vacuum The composite electrodes were assembled in 2032 coin cells using lithium foil as both counter and pseudo-reference electrode, and Celgard 2400 separator soaked in a 45:55 mixture of ethylenecarbonate and dmiethyl carbonate containing $1 \mathrm{M} \mathrm{LiPF}_{6}$ as electrolyte. All cell assembly and sample manipulation was performed in an Arfilled glovebox. A Bio-Logic VMP3 potentiostat/galvanostat were used to carry out all electrochemical experiments in galvanostatic mode, at $\mathrm{C} / 10$ rate. Capacity at the first discharge of the preliminary cell are in good agreements with the literatures $\left(156 \mathrm{mAh} \mathrm{g}^{-1}\right)$, showing that almost all particles were involved in the electrochemical reaction (Supplementary Fig. 1). The half-charged cell $(\sim 50 \%$ state-ofcharge) for the imaging experiments was stopped at to $78 \mathrm{mAh} \mathrm{g}^{-1}$ (Supplementary Fig. 1). 
Soft X-ray ptychographic microscope. Soft X-ray ptychographic microscopy measurements were performed at the bending magnet beamline (5.3.2.1) at the Advanced Light Source (ALS), Lawrence Berkeley National Laboratory ${ }^{11}, 12$. Ptychographic measurements utilized a $100 \mathrm{~nm}$ outer zone width Fresnel zone plate for illumination and proceeded with a square scan grid of $70 \mathrm{~nm}$ steps. Diffraction patterns from $200 \mathrm{~ms}$ exposure were directly recorded on a custom fast readout CCD with the 5- $\mu \mathrm{m}$-think $\mathrm{Si}_{3} \mathrm{~N}_{4}$ attenuator to expand the dynamic range (Supplementary Fig. 4). The diffraction data were reconstructed by 500 iterations of an implementation of the RAAR algorithm ${ }^{50}$. Incoherent background noise was eliminated through the implementation of a background retrieval algorithm ${ }^{11}$. All data processing, including pthychographic reconstruction, and background retrieval were performed using standard methods available in the SHARPCAMERA software package with parallel computation (http://camera.lbl.gov). The resolution of the individual $2 \mathrm{D}$ projection is calculated by Fourier ring correlation (FRC) to be $10 \mathrm{~nm}$ (1/2 bit threshold) at $708.2 \mathrm{eV}$ (Supplementary Fig. 6).

Registration of the rotation axis. Aligning the $2 \mathrm{D}$ projections of a tomographic tilt series to a common rotation axis (not necessarily the real rotation axis) with sub-pixel resolution is essential to achieve a good quality 3D reconstruction. In order to achieve sub-pixel-precision, we have developed an iterative registration method with intensity-base automatic image alignments. To set the common rotation axis, the projections were first roughly aligned using an alignment feature only with translations of pixel size. The alignment features in all roughly aligned projections were close to the common rotation axis, but there still exist huge misalignments owing to inaccuracy of human interactions and tilting of each projection. We then reconstructed the 3D volume from the first aligned tomographic tilt series and computed $2 \mathrm{D}$ projections of the $3 \mathrm{D}$ volume according to the tomographic tilt angles. These computed 2D projections were used as reference images for second alignments. The second alignment was performed with intensity-based automatic image registration, which is an iterative process brings the misaligned image (2D projections of the tomographic tilt series) into alignment with the reference image (computed $2 \mathrm{D}$ projections). The process was performed following non-reflective similarity transformations (consisting of translation, rotation, and scale) to determine the specific-image transformation matrix that is applied to the moving image with bilinear interpolation. The same procedures were repeated until the aligned projections were self-consistent.

Tomographic reconstruction. Tomographic imaging proceeded from a series of $1582 \mathrm{D}$ projections of the sample ODs recorded over a wide angular range from $-80^{\circ}$ to $+77^{\circ}$. After the image registration, the OD volumes at 708.2 and $710.2 \mathrm{eV}$ (Fig. 1a), with voxel size of $6.7 \times 6.7 \times 6.7 \mathrm{~nm}^{3}$, were reconstructed using the algebraic reconstruction technique (ART) with 20 iterations ${ }^{51}$. The $3 \mathrm{D}$ resolution is confirmed by FSC of the OD volume at $708.2 \mathrm{eV}$ and indicates a 3D spatial resolution around $11 \mathrm{~nm}$ (see Fig. $1 \mathrm{~b}$ and Supplementary Methods). A small improvement in the 3D resolution were observed by adopting different reconstruction algorithm with a large number of iterations, but the discrepancy did not affect the conclusions of the analysis (Supplementary Fig. 15). This value is confirmed by line-cuts through the volume, shown in Fig. 1c-d.

Chemical phase quantification. The OD volumes at 708.2 and $710.2 \mathrm{eV}$ were used for estimating quantitative chemical information (e.g., the oxidation state of iron in $\mathrm{Li}_{x} \mathrm{FePO}_{4}$ ) at each voxel. From the standard spectra of the discharged $\mathrm{Li}_{\alpha} \mathrm{FePO}_{4}$ (majority $\mathrm{Fe}^{2+}$ ) and charged $\mathrm{Li}_{\beta} \mathrm{FePO}_{4}$ (majority $\mathrm{Fe}^{3+}$ ), the relative absorption intensity $\left(I_{\mathrm{E} 1, \mathrm{LFP}}, I_{\mathrm{E} 1, \mathrm{FP}}, I_{\mathrm{E} 2, \mathrm{LFP}}\right.$, and $\left.I_{\mathrm{E} 2, \mathrm{FP}}\right)$ at specific energy $\left(E_{1}\right.$ and $\left.E_{2}\right)$ were acquired (Supplementary Fig. 5). Since, the amount of the absorption at a certain energy is linearly proportional to the relative amount of species with different iron oxidation states, the chemical concentration of $\mathrm{Li}_{\alpha} \mathrm{FePO}_{4}\left(\mathrm{C}_{\mathrm{LFP}}\right)$ and $\mathrm{Li}_{\beta} \mathrm{FePO}_{4}$ $\left(\mathrm{C}_{\mathrm{FP}}\right)$ at each voxel can be calculated by the relation:

$$
\left(\begin{array}{c}
\mathrm{OD}_{E 1}-\mathrm{OD}_{\text {pre-edge }} \\
\mathrm{OD}_{E 2}-\mathrm{OD}_{\text {pre-edge }}
\end{array}\right)=\left(\begin{array}{cc}
I_{E 1, \mathrm{LFP}} & I_{E 1, \mathrm{FP}} \\
I_{E 2, \mathrm{LFP}} & I_{E 2, \mathrm{FP}}
\end{array}\right)\left(\begin{array}{c}
C_{\mathrm{LFP}} \\
C_{\mathrm{FP}}
\end{array}\right)
$$

where $\mathrm{OD}_{E 1}, \mathrm{OD}_{E 2}$, and $\mathrm{OD}_{\text {pre-edge }}$ indicate the single voxel $\mathrm{OD}$ at $E_{1}, E_{2}$, and preedge region, respectively. While normalization of all ODs with $\mathrm{OD}_{\text {pre-edge }} \mathrm{can}$ maximize the chemical contrast, because $\mathrm{OD}_{\text {pre-edge }}$ is proportional to pure mass thickness without chemical contrast, the OD at pre-edge region was not clear enough to reconstruct 3D volume and negligible compared with ODs at 708.2 and $710.2 \mathrm{eV}$ (Supplementary Fig. 9). Since the concentration of each chemical phase, $\mathrm{LiFePO}_{4}\left(\mathrm{Fe}^{2+}\right)$ and charged $\mathrm{FePO}_{4}\left(\mathrm{Fe}^{3+}\right)$, can be expressed as linear equations corresponding to the OD volumes at 708.2 and $710.2 \mathrm{eV}$, the polar angle in the correlation plot is a function of the relative compositions of two major elements for the corresponding voxel (Fig. 2b). As a result, the 3D distribution of Fe oxidation state can be retrieved quantitatively (Fig. 2c). The fidelity of the 3D chemical map obtained in this way is verified by projecting the calculated volume along the $z$-axis and comparing with a map obtained by a linear combination fit of the reference spectra to independently measured 2D XAS data across the full spectrum of the same sample (Supplementary Figs. 11-12). The accuracy of the chemical map from 2D XAS data is represented by $R$-factor, which is less than 0.15 in $94.64 \%$ of pixels (Supplementary Fig. 10).
Data availability. The data that support the findings of this study are available from the corresponding author (D.A.S. or J.C.) on request.

Received: 12 February 2017 Accepted: 11 February 2018

Published online: 02 March 2018

\section{References}

1. Whittingham, M. S. Ultimate limits to intercalation reactions for lithium batteries. Chem. Rev. 114, 11414-11443 (2014).

2. Whittingham, M. S. Lithium batteries and cathode materials. Chem. Rev. 104, 4271-4302 (2004)

3. Miao, J., Ishikawa, T., Robinson, I. K. \& Murnane, M. M. Beyond crystallography: diffractive imaging using coherent $\mathrm{x}$-ray light sources. Science 348, 530-535 (2015).

4. Sakdinawat, A. \& Attwood, D. Nanoscale X-ray imaging. Nat. Photonics 4, 840-848 (2010).

5. Chao, W., Harteneck, B. D., Liddle, J. A., Anderson, E. H. \& Attwood, D. T. Soft X-ray microscopy at a spatial resolution better than $15 \mathrm{~nm}$. Nature $\mathbf{4 3 5}$, 1210-1213 (2005).

6. Delacourt, C., Poizot, P., Tarascon, J.-M. \& Masquelier, C. The existence of a temperature-driven solid solution in $\mathrm{Li}_{x} \mathrm{FePO}_{4}$ for $0 \leq x \leq 1$. Nat. Mater. 4, 254-260 (2005).

7. Pennycook, S. J. \& Boatner, L. A. Chemically sensitive structure-imaging with a scanning transmission electron microscope. Nature 336, 565-567 (1988).

8. Browning, N. D., Chisholm, M. F. \& Pennycook, S. J. Atomic-resolution chemical analysis using a scanning transmission electron microscope. Nature 366, 143-146 (1993).

9. Batson, P. E., Dellby, N. \& Krivanek, O. L. Sub-angstrom resolution using aberration corrected electron optics. Nature 418, 617-620 (2002).

10. Weyland, M. \& Midgley, P. A. 3D electron microscopy in the physical sciences: the development of Z-contrast and EFTEM tomography. Ultramicroscopy 96, 413-431 (2003).

11. Shapiro, D. A. et al. Chemical composition mapping with nanometre resolution by soft X-ray microscopy. Nat. Photonics 8, 765-769 (2014).

12. Yu, Y.-S. et al. Dependence on crystal size of the nanoscale chemical phase distribution and fracture in $\mathrm{Li}_{x} \mathrm{FePO}_{4}$. Nano. Lett. 15, 4282-4288 (2015).

13. Johansson, G. A., Tyliszczak, T., Mitchell, G. E., Keefe, M. H. \& Hitchcock, A. P. Three-dimensional chemical mapping by scanning transmission X-ray spectromicroscopy. J. Synchrotron Radiat. 14, 395-402 (2007).

14. Meirer, F. et al. Three-dimensional imaging of chemical phase transformations at the nanoscale with full-field transmission X-ray microscopy. J. Synchrotron Radiat. 18, 773-781 (2011).

15. Yang, F. et al. Nanoscale morphological and chemical changes of high voltage lithium-manganese rich NMC composite cathodes with cycling. Nano. Lett. 14, 4334-4341 (2014).

16. Padhi, A. K., Nanjundaswamy, K. S. \& Goodenough, J. B. Phospho-olivines as positive-electrode materials for rechargeable lithium batteries. J. Electrochem. Soc. 144, 1188-1194 (1997).

17. Zaghib, K., Mauger, A. \& Julien, C. M. Overview of olivines in lithium batteries for green transportation and energy storage. J. Solid State Electrochem. 16, 835-845 (2012).

18. Laffont, L. et al. Study of the $\mathrm{LiFePO}_{4} / \mathrm{FePO}_{4}$ two-phase system by highresolution electron energy loss spectroscopy. Chem. Mater. 18, 5520-5529 (2006).

19. Delmas, C., Maccario, M., Croguennec, L., Le Cras, F. \& Weill, F. Lithium deintercalation in $\mathrm{LiFePO}_{4}$ nanoparticles via a domino-cascade model. Nat. Mater. 7, 665-671 (2008).

20. Andersson, A. S. \& Thomas, J. O. The source of first-cycle capacity loss in $\mathrm{LiFePO}_{4}$. J. Power Sources 97-98, 498-502 (2001).

21. Malik, R., Zhou, F. \& Ceder, G. Kinetics of non-equilibrium lithium incorporation in $\mathrm{LiFePO}_{4}$. Nat. Mater. 10, 587-590 (2011).

22. Liu, H. et al. Capturing metastable structures during high-rate cycling of $\mathrm{LiFePO}_{4}$ nanoparticle electrodes. Science 344, 1252817 (2014).

23. Orikasa, Y. et al. Direct observation of a metastable crystal phase of $\mathrm{Li}_{x} \mathrm{FePO}_{4}$ under electrochemical phase transition. J. Am. Chem. Soc. 135, 5497-5500 (2013).

24. Zhang, X. et al. Rate-induced solubility and suppression of the first-order phase transition in olivine $\mathrm{LiFePO}_{4}$. Nano. Lett. 14, 2279-2285 (2014).

25. Liu, J., Kunz, M., Chen, K., Tamura, N. \& Richardson, T. J. Visualization of charge distribution in a lithium battery electrode. J. Phys. Chem. Lett. 1, 2120-2123 (2010).

26. Castro, L. et al. Aging mechanisms of $\mathrm{LiFePO}_{4} / /$ graphite cells studied by XPS: redox reaction and electrode/electrolyte interfaces. J. Electrochem. Soc. 159, A357-A363 (2012). 
27. Yamada, A. et al. Room-temperature miscibility gap in $\mathrm{Li}_{x} \mathrm{FePO}_{4}$. Nat. Mater. 5, 357-360 (2006).

28. Wagemaker, M. et al. Dynamic solubility limits in nanosized olivine $\mathrm{LiFePO}_{4}$. J. Am. Chem. Soc. 133, 10222-10228 (2011).

29. Hess, M., Sasaki, T., Villevieille, C. \& Novák, P. Combined operando X-ray diffraction-electrochemical impedance spectroscopy detecting solid solution reactions of LiFePO4 in batteries. Nat. Commun. 6, 8169 (2015).

30. Wang, L. et al. Crystal orientation tuning of $\mathrm{LiFePO}_{4}$ nanoplates for high rate lithium battery cathode materials. Nano. Lett. 12, 5632-5636 (2012).

31. Miao, S. et al. Local electronic structure of olivine phases of $\mathrm{Li}_{x} \mathrm{FePO}_{4}$. J. Phys. Chem. A. 111, 4242-4247 (2007).

32. Augustsson, A. et al. Electronic structure of phospho-olivines $\mathrm{Li}_{x} \mathrm{FePO}_{4}(x=$ $0,1)$ from soft-x-ray-absorption and -emission spectroscopies. J. Chem. Phys. 123, 184717 (2005).

33. Liu, X. et al. Phase transformation and lithiation effect on electronic structure of $\mathrm{Li}_{x} \mathrm{FePO}_{4}$ : an in-depth study by soft x-ray and simulations. J. Am. Chem. Soc. 134, 13708-13715 (2012).

34. Li, Y. et al. Dichotomy in the lithiation pathway of ellipsoidal and platelet $\mathrm{LiFePO}_{4}$ particles revealed through nanoscale operando state-of-charge imaging. Adv. Funct. Mater. 25, 3677-3687 (2015).

35. Kao, T. L. et al. Nanoscale elemental sensitivity study of $\mathrm{Nd}_{2} \mathrm{Fe}_{14} \mathrm{~B}$ using absorption correlation tomography. Microsc. Res. Tech. 76, 1112-1117 (2013).

36. Lim, J. et al. Origin and hysteresis of lithium compositional spatiodynamics within battery primary particles. Science 353, 566-571 (2016).

37. Ramana, C. V., Mauger, A., Gendron, F., Julien, C. M. \& Zaghib, K. Study of the $\mathrm{Li}$-insertion/extraction process in $\mathrm{LiFePO}_{4} / \mathrm{FePO}_{4}$. J. Power Sources 187, 555-564 (2009).

38. Suo, L. et al. Highly ordered staging structural interface between $\mathrm{LiFePO}_{4}$ and $\mathrm{FePO}_{4}$. Phys. Chem. Chem. Phys. 14, 5363-5367 (2012).

39. Zhou, F., Maxisch, T. \& Ceder, G. Configurational electronic entropy and the phase diagram of mixed-valence oxides: the case of $\mathrm{Li}_{x} \mathrm{FePO}_{4}$. Phys. Rev. Lett. 97, 155704 (2006).

40. Zhang, X. et al. Direct view on the phase evolution in individual $\mathrm{LiFePO}_{4}$ nanoparticles during Li-ion battery cycling. Nat. Commun. 6, 8333 (2015).

41. Wagemaker, M., Borghols, W. J. H. \& Mulder, F. M. Large impact of particle size on insertion reactions. a case for anatase $\mathrm{Li}_{x} \mathrm{TiO}_{2}$. J. Am. Chem. Soc. 129, 4323-4327 (2007).

42. Dreyer, W. et al. The thermodynamic origin of hysteresis in insertion batteries. Nat. Mater. 9, 448-453 (2010).

43. Chueh, W. C. et al. Intercalation pathway in many-particle $\mathrm{LiFePO}_{4}$ electrode revealed by nanoscale state-of-charge mapping. Nano. Lett. 13, 866-872 (2013).

44. Li, Y. et al. Current-induced transition from particle-by-particle to concurrent intercalation in phase-separating battery electrodes. Nat. Mater. 13 1149-1156 (2014).

45. Brunetti, G. et al. Confirmation of the domino-cascade model by $\mathrm{LiFePO}_{4} /$ $\mathrm{FePO}_{4}$ precession electron diffraction. Chem. Mater. 23, 4515-4524 (2011).

46. Welland, M. J., Karpeyev, D., O'Connor, D. T. \& Heinonen, O. Miscibility gap closure, interface morphology, and phase microstructure of $3 \mathrm{D} \mathrm{Li}_{x} \mathrm{FePO}_{4}$ nanoparticles from surface wetting and coherency strain. ACS Nano 9, 9757-9771 (2015).

47. Cogswell, D. A. \& Bazant, M. Z. Coherency strain and the kinetics of phase separation in $\mathrm{LiFePO}_{4}$ nanoparticles. ACS Nano 6, 2215-2225 (2012).

48. Abdellahi, A., Akyildiz, O., Malik, R., Thorntonc, K. \& Ceder, G. The thermodynamic stability of intermediate solid solutions in $\mathrm{LiFePO}_{4}$ nanoparticles. J. Mater. Chem. A 4, 5436-5447 (2016).

49. Cogswell, D. A. \& Bazant, M. Z. Theory of coherent nucleation in phaseseparating nanoparticles. Nano. Lett. 13, 3036-3041 (2013).

50. Luke, D. R. Relaxed averaged alternating reflections for diffraction imaging. Inverse Probl. 21, 37 (2005).
51. Gordon, R., Bender, R. \& Herman, G. T. Algebraic Reconstruction Techniques (ART) for three-dimensional electron microscopy and X-ray photography. J. Theor. Biol. 29, 471-481 (1970).

\section{Acknowledgements}

Soft X-ray ptychographic microscopy was carried out at beamline 5.3.2.1 at the Advanced Light Source. The Advanced Light Source is supported by the Director, Office of Science, Office of Basic Energy Sciences, of the U.S. Department of Energy under Contract No. DE-AC02-05CH11231. This work was supported as part of the NorthEast Center for Chemical Energy Storage, an Energy Frontier Research Center funded by the U.S. Department of Energy, Office of Science, Office of Basic Energy Sciences under Award Number DE-SC0012583. C.K. acknowledges additional support by the National Research Lab (NRF-2015R1A2A1A01006192) program of the National Research Foundation of Korea. This work is partially supported by the Center for Applied Mathematics for Energy Research Applications (CAMERA), which is a partnership between Basic Energy Sciences (BES) and Advanced Scientific Computing Research (ASRC) at the U.S Department of Energy.

\section{Author contributions}

Y.-S.Y., C.K., J.C., and D.A.S. conceived of and planned the experiment. D.A.S., T.T., R.C., P.D., J.J., H.K., F.R.N.C.M., A.L.D.K., T.P.C.L., T.W., Y.-S.Y., and H.P. developed experimental techniques, software, and equipment. D.A.S. and S.M. developed ptychographic reconstruction codes. Y.-S.Y., C.K., F.C.S., and C.P.G. prepared the samples. Y.-S.Y., M.F., and D.A.S. carried out the ptychographic microscopy measurements. Y.-S.Y., Y.L., and D.A.S. performed post-experiment data analysis, and Y.-S.Y., Y.L., C.K., D.A.S., and J.C. established the interpretation of the chemical maps. Y.-S.Y., C.K., J.C., and D.A.S. prepared the manuscript, which incorporates critical input from all authors

\section{Additional information}

Supplementary Information accompanies this paper at https://doi.org/10.1038/s41467 018-03401-x.

Competing interests: The authors declare no competing interests.

Reprints and permission information is available online at http://npg.nature.com/ reprintsandpermissions/

Publisher's note: Springer Nature remains neutral with regard to jurisdictional claims in published maps and institutional affiliations.

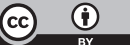

Open Access This article is licensed under a Creative Commons Attribution 4.0 International License, which permits use, sharing, adaptation, distribution and reproduction in any medium or format, as long as you give appropriate credit to the original author(s) and the source, provide a link to the Creative Commons license, and indicate if changes were made. The images or other third party material in this article are included in the article's Creative Commons license, unless indicated otherwise in a credit line to the material. If material is not included in the article's Creative Commons license and your intended use is not permitted by statutory regulation or exceeds the permitted use, you will need to obtain permission directly from the copyright holder. To view a copy of this license, visit http://creativecommons.org/ licenses/by/4.0/.

(c) The Author(s) 2018 